\title{
Photonic crystal electrode to be used in organic LED structures
}

L. Petti

\section{Rippa \\ R. Capasso}

G. Nenna

giuseppe.nenna@enea.it

\section{A. De Girolamo Del Mauro}

\section{La Ferrara}

\section{A. Pacheri Madathil}

\section{Minarini}

Cybernetics Institute of CNR, 80072 Pozzuoli, Italy

Cybernetics Institute of CNR, 80072 Pozzuoli, Italy

Cybernetics Institute of CNR, 80072 Pozzuoli, Italy

UTTP ENEA Portici Research Centre, 80055 Portici, Italy

UTTP ENEA Portici Research Centre, 80055 Portici, Italy

UTTP ENEA Portici Research Centre, 80055 Portici, Italy

UTTP ENEA Portici Research Centre, 80055 Portici, Italy

UTTP ENEA Portici Research Centre, 80055 Portici, Italy

In this work we report the possibility to obtain a high refractive index grid anode directly on the substrate surface by fabricating a relatively large-area photonic crystal (PC) structure using the combinations of electron beam lithography (EBL) and focused ion beam (FIB) techniques. The performance of the realized photonic crystal (PC) structure were enhanced by milling the ITO layer until the glass substrate and by removing the further refractive index jump between the PC and the substrate. The good properties of highly conductive poly (3,4 ethylenedioxythiophene):poly(styrene sulfonate) (PEDOT:PSS), ensured a continuous path for the current and a high refractive index jump for the PC structure by filling the holes in the PC structure.

[DOI: http://dx.doi.org/10.2971/jeos.2013.13002]

Keywords: OLED, photonic crystal, efficiency, light trapping

\section{INTRODUCTION}

The organic light emitting diode (OLED) can play a key role in the new frontier of lighting and one of the remarkable notes of this technology is the energy savings that may result from their employment. One of the essential points to ensure the development of this technology is the devices external quantum efficiency.

The external quantum efficiency of conventional devices is around $20 \%$ because of losses due to wave-guiding effect. Recently, there has been great progress to enhance the light outcoupling efficiency of organic electroluminescent devices by means of various internal and external device modification techniques. Several strategies were developed concerning the surface modification to increase the efficiency factor by using ordered micro-lenses, scattering layers, bragg reflectors, 2D photonic crystal or modifying the cavity effects [1]-[4].

As far as the jump in the refractive index at TCO (Transparent Conductive Oxide) - substrate interface many suggestions and directions can be found in literature. Technological solutions require the introduction of an additional layer between the TCO and the substrate. Y. Sun and R. Forrest demonstrated in 2008 that embedding a low-index grid in the organic layers can enhance the out-coupling of the waveguided light with an improvement of the external quantum efficiency of a white OLED to $34 \%$ [5]. A further application makes use of wrinkling the emissive area: even a doubling in the performance can be obtained due to a Bragg scattering effect [6]. Another technique makes use of a two-dimensional nanopatterning to create a photonic crystal (PC) which improves substantially the outcoupled light [7]-[9].

The 2D-PC OLEDs exhibit remarkable enhancement in electroluminescent (EL) intensity in the forward direction with the optical outcoupling structures usually located between the OLED stack and the substrate to bring more light into the substrate which finally leads to a higher amount of photons extracted into the air. To simplify the device architecture of 2DPC OLEDs, a first effort on partially structured ITO electrode was realized by Fujita et al [10] which showed an improvement in efficiency due to the enhancement of light extraction efficiency for confined modes in ITO/organic layers by photonic crystal effect. More recently, ITO electrode was partially patterned by focused ion beam (FIB) covered by PEDOT:PSS 
finding a good increasing of efficiency [11]. The relative low refractive index of PEDOT:PSS [12] gives a high refractive index jump for the ITO-PEDOT:PSS structure that is advantageous also on microstructured grid [13].

Moreover, to ensure a wider range of applications and to lower significantly the prices of production to OLEDs application, many efforts have been focused on improvement of electrical conductivity of conjugated conductive polymers, especially of the poly(3,4-ethylenedioxythiophene):poly(styrene sulfonate) (PEDOT:PSS) films. Electrical conductivity of PEDOT:PSS can be enhanced by acidic and thermal treatments. Improvement was achieved by adding small amount of solvents with high boiling point such as dimethyl formamide (DMF), dimethyl sulfoxide (DMSO), glycerol, and sorbitol, etc., reaching considerable conductivity values $(\sigma>700 \mathrm{~S} / \mathrm{cm})[14,15]$.

In this work we show how it is possible to nanostructure completely on the ITO electrode using electron beam lithography (EBL)/focused ion beam (FIB) combination. The use of these two techniques allows to realize ITO grid having a high index grid embedded in a relatively large-area photonic crystal (PC) structure. The fabricated PC-structure could be more advantageous than partially structured ITO electrode because the ITO was entirely milled and thus increasing the photon extraction of the final structure. The highly conductive PEDOT:PSS was used as a continuous path for the current by filling the holes and obtaining a high refractive index jump.

\section{EXPERIMENTAL}

Device structures were fabricated by using high resolution electron beam lithography (EBL) and focused ion beam (FIB) techniques. The EBL facility consists of a Raith 150 system, capable of writing patterns of arbitrary geometries with a spatial resolution up to $10 \mathrm{~nm}$. The samples were obtained by exposing a layer of ZEP deposited on an indium tin oxide (ITO) coated glass. The e-beam is locally focused on the sample to expose selected regions of material homogenously along the depth of the substrate according to the calculated desired pattern. The resulting two dimensional photonic crystal is made of air rods embedded into the organic matrix of ZEP. The air rods arrays were fabricated using EBL on glass substrate with a $120 \mathrm{~nm}$ layer of ITO for conduction. A $200 \mathrm{~nm}$-thick layer of ZEP was spin coated on top of the cleaned substrate. Subsequently, the nanopatterning was defined using the Raith 150 system with current and area dosage of $23.6 \mathrm{pA}$ at $10 \mathrm{KeV}$. The resist was developed in n-Amyl acetate.

Thus, a 2D-PC pattern with holes of $160 \mathrm{~nm}$ and period of $275 \mathrm{~nm}$ has been uniformly fabricated by EBL on a surface of around $1.5 \times 1.5 \mathrm{~mm}^{2}$ (see Figure 1). The PC was realized taking into account the relationship between the guided light spectrum and Braggs diffraction condition.

A focused ion beam (FEI Quanta 200 3D), composed by gallium ions with an energy of $30 \mathrm{kV}$, was then used for milling of large area PC structure realizing holes into the ITO layer using the structured ZEP-520 as a lithographic mask. FIB working

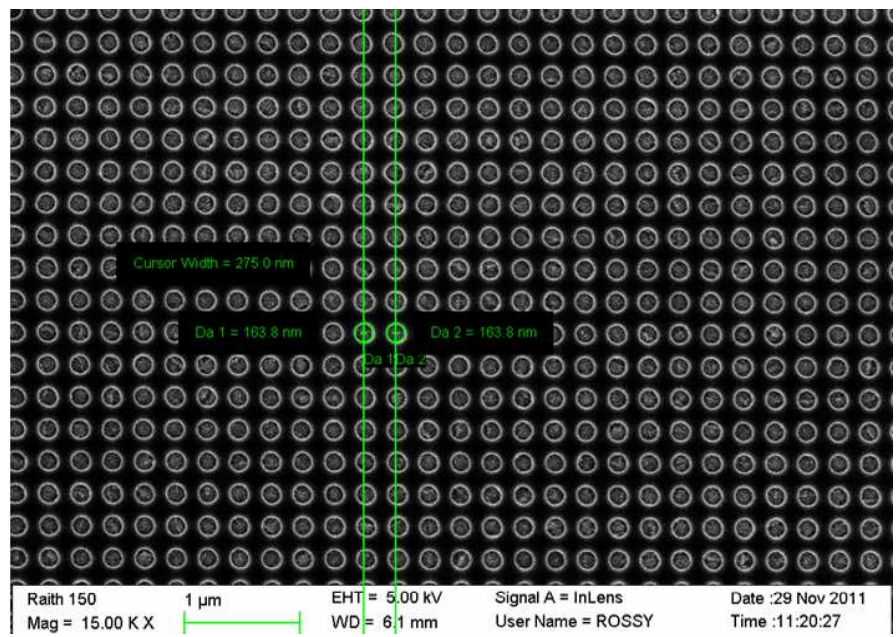

FIG. 1 SEM image of the 2D PCS obtained by arranging air rods into the ZEP (diameter $d=160 \mathrm{~nm}$ and lattice constant $a=275 \mathrm{~nm}$ ).

conditions were optimized to control the ITO milling process until the glass-substrate. This was achieved by controlling the sample absorbed current when gallium ions strike the surface. In fact secondary electron and secondary ion signals are material dependent and provide indications of milling the interface between the different materials which form the PC structure [16] by detecting the end point monitor. In order to mill a large area of PC, FIB beam current of $20 \mathrm{nA}$ at an accelerating voltage of $30 \mathrm{kV}$ was used. By means of these working conditions and modifying the beam overlap, it was possible to mill an area of $0.4 \mathrm{~mm}^{2}$ in a relative fast time. The ZEP was subsequently removed by high-vacuum PECVD multi-chamber system by plasma etching [17] realized by an ELETTRORAVA multi-chamber with these parameters: power around $30 \mathrm{~W}$, $30 \mathrm{sccm}$ for the $\mathrm{O}_{2}$, and pressure around 0.25 Torr for $8 \mathrm{~min}-$ utes. This also ensures a good ITO treatment and a good adhesion for the PEDOT:PSS conductive layer.

After removing the resist highly conductive PEDOT:PSS doped by DMSO was deposited by filling the holes, realizing a continuous path for the current and obtaining a high refractive index jump for the PC structure. The process steps and the related schematic structures are summarized in the Figure 2.

Finally an optical setup was adopted to evaluate the PC properties and to estimate the OLED spectrum properties. The setup used to measure the light propagating in the glass substrate by extracting the diffraction is reported in Figure 3 and is composed of a multimode fiber, a CCD imaging telescope (OL610), and a CCD-based spectroradiometer (OL770-LED). Finally, to test the possibility of using this type of PC structure in an OLED device, a staked device was realized on the structured area. The structure of OLED device under investigation was ITO (anode) / PEDOT:PSS / Poly(9,9-di-n-octylfluorenyl2,7-diyl)(PFO) / Calcium-Aluminium (cathode). The devices were fabricated on structured and unstructured ITO surface of the same substrate to prevent changes due to different growth conditions. current-electroluminescence (I-EL) characteristics have been measured using a Keithley 2400 power supply source meter with constant increment steps and delay time of $1 \mathrm{~s}$ before each measurement point. Electrolumines- 


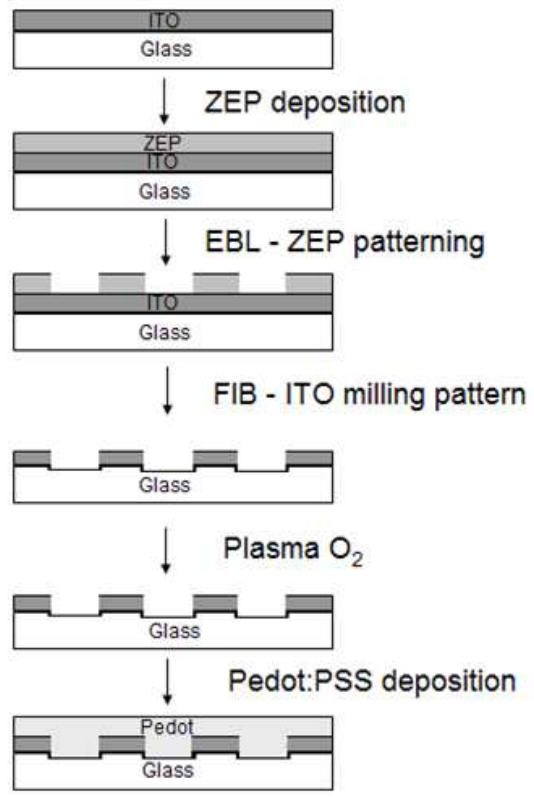

FIG. 2 Schematic diagram of process for ITO-PEDOT:PSS PC realization.

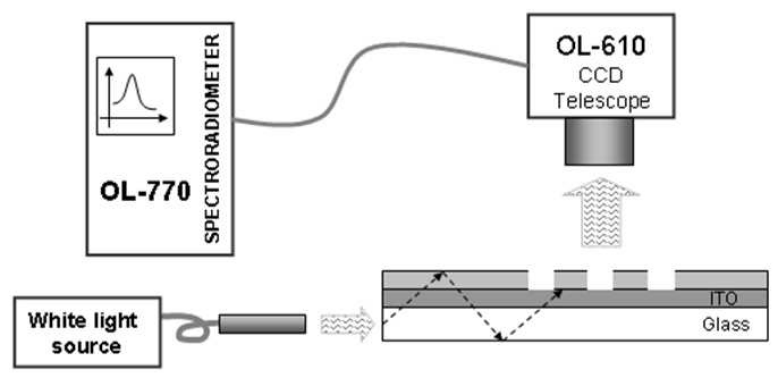

FIG. 3 Optical set up employed for the PC evaluation and spectra measurements.

cence analysis was performed using a photodiode (Newport 810UV) connected to a Keithley 6517A Electrometer. The device electroluminescence spectra were evaluated through an Ocean-Optics USB4000 spectrometer.

\section{RESULTS AND DISCUSSION}

After the EBL lithography step the device was tested by introducing a white light from the edge of the glass substrate that propagated inside the glass itself due to the total internal reflection phenomena, as shown in Figure 3. The PC area was seen as a blue area because of the wavelength-selective diffraction by the hybrid PC. Figure 4 shows spectrally integrated light intensity characteristics of the sample as detected normally from the sample surface compared to simulated results. The resonant peak appears due to the PC feedback effect, and the full width at half maximum of spectrum is of $10 \mathrm{~nm}$ at the main peak, that is located around $400 \mathrm{~nm}$. The same experiment was also performed on the structured surface after the FIB milling, showing a red shift of around $20 \mathrm{~nm}$. The peak spectrum was in perfect agreement with the simulated spectrum employing a finite-difference time-domain numerical method (FDTD) performed with commercial software (Full Wave simulation tool, RSoft Design Group, Ossining, NY). The time step (in unit of ct) used during the simulation was $66 \times 10^{-4} \mu \mathrm{m}$. In both calculations geometric parame-

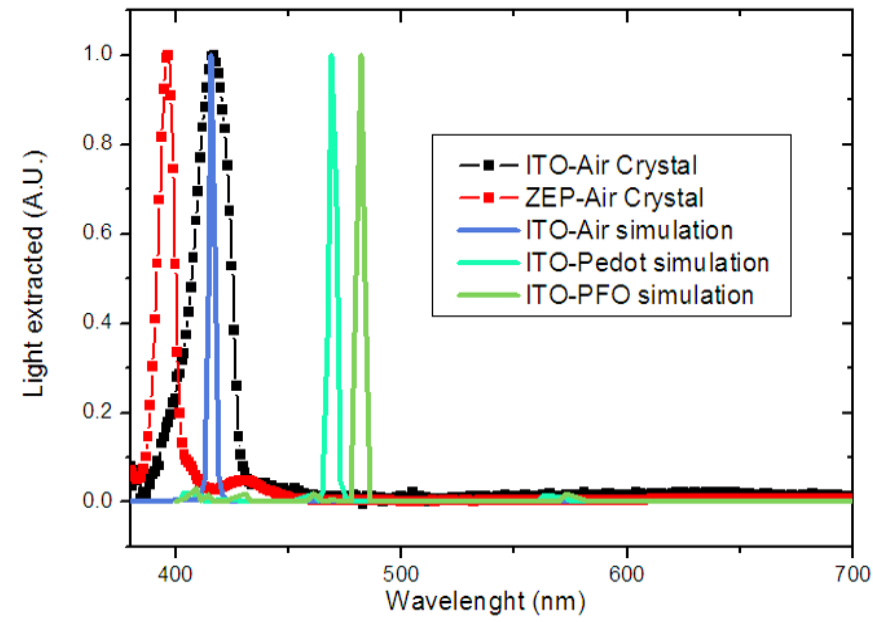

FIG. 4 Measured spectrum of the fabricated sample. In the graph the spectrum coming from the ZEP crystal and the ITO crystal after FIB milling step are reported. Moreover the evaluation of the spectra coming from an ITO crystal, ITO-PEDOT and ITO-PFO structures are reported.

ters referring to a single square unit cell were used. In order to simulate a periodic structure periodic boundary conditions on the $\mathrm{x}-\mathrm{z}$ plane, and perfectly matched layer boundary condition on y direction were used. The structures were excited from the air by a short impulse with a broad spectrum and plane wave front. For simplicity, it is assumed that the glass substrate (corning eagle 2000) and air are semi-infinite with a constant refractive index $n_{\text {glass }}=1.51$ and $n_{\text {air }}=1$. For the ITO/glass structure the period was $a=270 \mathrm{~nm}$, radius of the air holes $r=82 \mathrm{~nm}$, thickness of the ITO $t_{\text {ITO }}=120 \mathrm{~nm}$ and depth of the holes $t_{\text {holes }}=120 \mathrm{~nm}$. In both calculations a spatial grid with step size of $10 \mathrm{~nm}$ for $\mathrm{x}-\mathrm{z}$ plane and 20 $\mathrm{nm}$ for $\mathrm{y}$ direction were used. The numerical domain which was used correspond to a supercell of dimensions $(1,4,1)$. To better explain the optical effect of the PC structure on the device spectrum, the same simulation were also performed on the ITO-PEDOT:PSS and on the ITO-PFO crystals. The refractive index used during the simulation was: $n_{1}(\mathrm{ITO})=1.9$, $n_{2}$ (Pedot:PSS $)=1.53$ and $n_{3}(\mathrm{PFO})=1.74$ [12].

In Figure 4, the emitted peaks during the experimental PC evaluation (see also Figure 3) together with the simulated results were reported. These resonant peaks are considered to originate from different transverse guiding modes with different modal indices, which are diffracted to the normal direction by the PC effect [18]. Here, Bragg-diffraction effects by the corrugated structure to improve light extraction efficiency were also considered. By utilizing the combination of several light waves by Bragg diffraction within the 2-D plane, it becomes possible to compose a stable wave state that extends throughout the entire plane [19]. Thus it is possible to obtain an oscillation mode, in which the electromagnetic distribution is fully controlled at each lattice point of the 2-D crystal [20].

After the ITO structuring, the studies were focused to improve the PEDOT:PSS performances. The employed PEDOT:PSS was recently developed and purchased from H. C. Starck (Baytron PH 1000). The concentration of PEDOT:PSS in water was $1-1.3 \%$ by weight with a weight ratio of PEDOT to PSS of 1:2.5. In order to enhance the conductivity of the 
pristine PEDOT:PSS, 5\% DMSO was added to the Baytron PH 1000 solution (hereafter, indicated as DMSO-PEDOT:PSS) and stirred for $24 \mathrm{~h}$ at room temperature. After filtration through a $0.45 \mathrm{~m}$ polyvinyl difluoride (PVDF) filter, the conductive polymer dispersions without and with DMSO were spin-coated onto glass and glass/ITO substrates. The deposited films were baked for $30 \mathrm{~min}$ at $100{ }^{\circ} \mathrm{C}$ in a vacuum oven. The sheet resistance values of the undoped PEDOT:PSS and DMSO-PEDOT:PSS films were $8.30 \times 10^{5} \mathrm{Ohm} / \mathrm{m}^{2}$ and $2.85 \times 10^{2} \mathrm{Ohm} / \mathrm{m}^{2}$, respectively. This indicates that there is a remarkable improvement of the electrical conductivity by adding 5\% DMSO to pristine dispersion, reaching the value of $700 \mathrm{~S} / \mathrm{cm}[14,15]$.

The DMSO doped PEDOT:PSS was spun onto the structured ITO realizing a peculiar PC anodic contact, ensuring a good refractive index jump [21] and a continuous current pathway. Finally Poly(9,9-di-n-octylfluorenyl-2,7-diyl)(PFO) powder was dissolved in toluene $(10 \mathrm{mg} / \mathrm{ml})$ and was spun onto PEDOT:PSS layer obtaining around $100 \mathrm{~nm}$ thick emitter layer. The device was completed with the calcium/aluminium cathode electrode, through thermal vacuum deposition with base pressure of $1 \times 10^{-8}$ Torr. The OLED device was tested under nitrogen atmosphere to prevent the calcium oxidation. Figure 5(a) shows the electroluminescence behaviour in terms of the photodiode current versus the device current and Figure 5 (b) shows the spectral modification due to the photonic crystal presence. From the first graph it is possible to have an idea of the efficiency improvement that can be evaluated to be a factor around 1.7. This is also observed on the spectral evaluations that suggest a spectral modification around $480 \mathrm{~nm}$ due to the PC pattern (see also Figure 4). The spectral modification could be ascribed to more than one cause and in particular probably also to the possible partial covering of the PEDOT:PSS layer that continue to ensure the current paths but give the possibilities to create more than one photon-escaping modes. Our results are due not only to the ITO-PEDOT crystal but also to the ITO-PFO crystal due to the PFO partial permeation into the ITO holes. It is found that the presented results are in good agreement with those reported in literature [22]-[25]. In particular, the PC-structure reported in this results turns out to be more advantageous than partially structured ITO electrode (improvement factor 1.46) [10] because the ITO was entirely milled and, in addition, became a high index grid onto the glass substrate [13] (improvement factor 1.42) increasing the photon extraction of the final structure. Moreover, in a corrugated OLED, the corrugation depth of the PC layers and the metal contact can be considered equal, but there are several possibilities to use planarization techniques to have a partial or complete planarization. This could be a critical point, indeed when the corrugation depth exceeds a certain value; deeper metal cavity causes random phased electromagnetic waves due to multi-reflection and diffraction from the metal surface and side walls. Energy absorption by the surface plasmon from the metal surface leads to the reduction of the light extraction as well. These factors reduce the amount of Bragg reflected light waves, resulting in less improvement of efficiency [26].

In this case with the PEDOT:PSS layer a partial planarization of PC structure can be obtained. For this reason, a deeper pillar
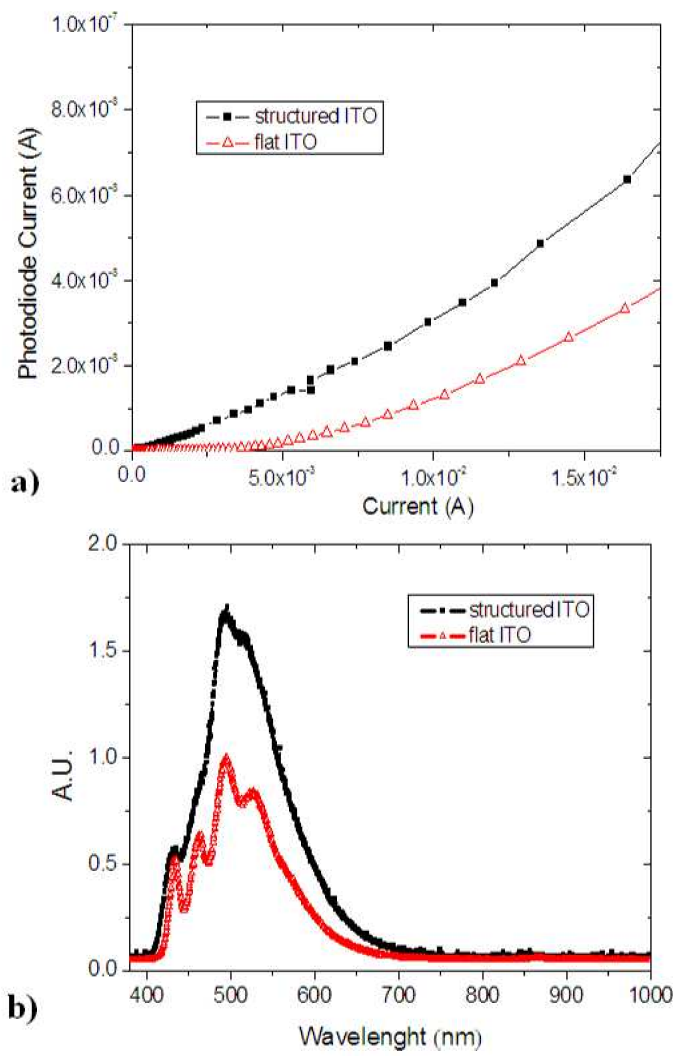

FIG. 5 (a) Photodiode current versus the device current of the OLED device. (b) Spectra related to structured and unstructured OLED devices droved at the same current (15 mA).

depth with a larger Bragg diffraction effect can be realized and it is possible to obtain a good Bragg reflection effect by combining the two effects in a proper way. By introducing the PEDOT:PSS layer it is possible to prevent the formation of cracks and sharp edges that can cause leakages current and a drastic efficiency reduction, vanishing the PC effect [27]. Finally, in this way it is not necessary to make further process steps to realize the photonic structure below the anode [28], by obtaining a good jump of refractive index useful for the release of trapped light $[13,29]$ and using a very thin TCO (50-100 nm), which in turn fosters a better optical coupling [30].

\section{CONCLUSIONS}

In conclusion, the possibility to realize a discontinuous refractive index path directly on the anode surface combining EBL and FIB processes and thereby enhancing the performance of the realized PC structure by removing a further refractive index jump between the PC and the substrate was demostrated. The low-index points realized by the presence of PEDOT:PSS could redirect modes normally trapped within the high-index organic and indium tin oxide layers that improve the OLED devices efficiency performance. Moreover the presence of the spun PEDOT:PSS will prevent the formation of cracks and sharp edges that can damage the device performances in this kind of applications. In this preliminary study the PC-ITO electrode guaranteed an improvement factor of around 1.7 in the efficiency. Thus this technique is a potential candidate for the enhancement of light extraction efficiency in OLEDs and it turns out to be more advantageous than partial PC or mi- 
crometric structuring of ITO electrode already reported in the literature.

\section{ACKN OWLEDGEMENTS}

Dr. Mariagrazia Maglione and Giuseppe Pandolfi at ENEA are acknowledged for the cathode evaporation process and the plasma etching treatment. The present work has been financially supported by Relight project PN: PON02_00556_3306937.

\section{References}

[1] S. Moller, and S. R. Forrest, "Improved light out-coupling in organic light emitting diodes employing ordered microlens arrays," J. Appl. Phys. 91, 3324 (2002).

[2] G. Nenna, A. De Girolamo Del Mauro, E. Massera, A. Bruno, T. Fasolino, and C. Minarini, "Optical Properties of Polystyrene-Zno Nanocomposite Scattering Layer to Improve Light Extraction in Organic Light-Emitting Diode," J. Nanomater. 2012, 319398 (2012).

[3] Y. J. Lee, S.-H. Kim, J. Huh, G.-H. Kim, Y.-H. Lee, S.-H. Cho, Y.-C. Kim, and Y. Rag Do, "A high-extraction-efficiency nanopatterned organic light-emitting diode," Appl. Phys. Lett. 82, 3779 (2003).

[4] W. Li, R. A. Jones, S. C. Allen, J. C. Heikenfeld, and A. J. Steck, “Maximizing Alq3 OLED internal and external efficiencies: charge balanced device structure and color conversion outcoupling lenses," J. Displ. Technol. 2-2, 143-152 (2006).

[5] Y. Sun, and S. R. Forrest, "Enhanced light out-coupling of organic light-emitting devices using embedded low-index grids," Nat. Photon. 2, 483-487 (2008).

[6] J. M. Lupton, B. J. Matterson, and I. D. W. Samuel, "Bragg scattering from periodically microstructured light emitting diodes," Appl. Phys. Lett. 77,(21), 3340-3342 (2000)

[7] S. Noda, M. Fujita, and T. Asano, "Spontaneous-emission control by photonic crystals and nanocavities," Nat. Photonics 1(8), 449-458 (2007).

[8] Y. Rong, and W. Qing, "High light-extraction-efficiency OLED based on photonic crystal slab structures with taper unit cells," Optoelectron. Lett. 2(2), 86-90 (2006).

[9] M. Boroditsky, T. F. Krauss, R. Coccioli, R. Vrijen, R. Bhat, and E. Yablonovitch, "Spontaneous emission extraction and purcell enhancement from thin-film 2-D photonic crystals," Appl. Phys. Lett. 75(8), 2096-2112 (1999).

[10] M. Fujita, T. Ueno, T. Asano, S. Noda, H. Ohhata, T. Tsuji, H. Nakada, and N. Shimoji, "Reduction of Operating Voltage in Organic Lightemitting Diode by Corrugated Photonic Crystal Structure," Electron. Lett. 39, 24 (2003).

[11] C. Tsai, L. Liao, Y. Luo, P. C. Chao, E. Chen, H. Meng, W. Chen, et al., "Optimal design and fabrication of ITO/organic photonic crystals in polymer light-emitting diodes using a focused ion beam," Microelectron. Eng. 87, 1331-1335 (2010).

[12] D. Zhu, W. Shen, H. Ye, X. Liu, and H. Zhen, "Determination of the optical constants of polymer light-emitting diode films from single," J. Phys. D: Appl. Phys. 41, 235104 (2008).

[13] T.-W. Koh, J.-M. Choi, S. Lee, and S. Yoo, "Optical outcoupling enhancement in organic light-emitting diodes: highly conductive polymer as a low-index layer on microstructured ITO electrodes," Adv. Mater. 21, 1-5 (2010).
[14] Y. Zhou, H. Cheun, S. Choi, W. J. Potscavage Jr., C. FuentesHernandez, and B. Kippelen, "Indium tin oxide-free and metal-free semitransparent organic solar cells," Appl. Phys. Lett. 97, 153304 (2010).

[15] A. De Girolamo Del Mauro, G. Nenna, F. Villani, and C. Minarini, "Study of the effect of the doped poly $(3,4$ ethylenedioxythiophene):poly(styrene sulfonate) polymeric anode on the organic light emitting diode performances," Thin Solid Films 520, 1653866 (2012).

[16] H. X. Qian, W. Zhou, X. M. Li, J. M. Miao, and L. E. N. Lim, “Appl. Time Dependent Morphological Transition and Sputtering Yield of Ion Sputtered Sn," Appl. Surf. Sci. 253, 5511-5515 (2007)

[17] F. D. Egitto, "Plasma etching and modification of organic polymers," Pure Appl. Chem. 62-9, 1699-1708 (1990).

[18] M. Fujita, K. Ishihara, T. Ueno, T. Asano, S. Noda, H. Ohata, T. Tsuji, H. Nakada, and N. Shimoji, "Optical and Electrical Characteristics of Organic Light-Emitting Diodes with Two-Dimensional Photonic Crystals in Organic/Electrode Layers," Jpn. J. Appl. Phys. 44, 3669-3677 (2005).

[19] M. Imada, S. Noda, A. Chutinan, T. Tokuda, M. Murata, and G. Sasaki, "Coherent two-dimensional lasing action in surfaceemitting laser with triangular-lattice photonic crystal structure," Appl. Phys. Lett. 75, 316-318 (1999).

[20] S. Noda, "Recent Progresses and Future Prospects of Two- and Three-Dimensional Photonic Crystals," J. Lightwave Technol. 24, 4554 (2006).

[21] L. A. A. Pettersson, S. Ghosh, and 0. Inganas, “Optical anisotropy in thin films of poly(3,4-ethylenedioxythiophene)poly(4-styrenesulfonate)," Org. Electron. 3, 143-148 (2002).

[22] M. Kitamura, S. Iwamoto, and Y. Aarakawa, "Enhanced luminance efficiency of organic light-emitting diodes with two-dimension photonic crystals," Jpn. J. Appl. Phys. 44-4B, 2844-2848 (2005).

[23] K. Ishihara, M. Fujita, I. Matsubara, T. Asano, and S. Noda, “Organic light-emitting diodes with photonic crystals on glass substrate fabricated by nanoimprint lithography," Appl. Phys. Lett. 90, 111114 (2007).

[24] Y.-C. Kim, S.-H. Cho, Y.-W. Song, Y.-J. Lee, Y.-H. Lee, and Y. Rag Do, "Planarized SiNx/spin-on-glass photonic crystal organic lightemitting diodes," Appl. Phys. Lett. 89, 173502 (2006).

[25] K. Saxena, V. K. Jain, D. Singh Mehta, "A review on the light extraction techniques in organic electroluminescent devices," opt. Mater. 32, 221-233 (2009).

[26] C. J. Yates, I. Samuel, P. Burn, S. Wedge, and W. L. Barnes, "Surface plasmon-polariton mediated emission from phosphorescent dendrimer light-emitting diodes," Appl. Phys. Lett. 88, 161105 (2006).

[27] A. 0. Altuna, S. Jeonb, J. Shima, J.-H. Jeonga, D.-G. Choia, K.-D. Kima, J.-H. Choi, et al., "Corrugated organic light emitting diodes for enhanced light extraction," Org. Electron. 11, 711-716 (2010).

[28] Y.-C. Kim, S.-H. Cho, Y.-W. Song, Y.-J. Lee, Y.-H. Lee, and Y. Rag, "Planarized SiNx/spin-on-glass photonic crystal organic lightemitting diodes," Appl. Phys. Lett. 89, 173502 (2006).

[29] M. Slootsky, and S. R. Forrest, "Full-wave simulation of enhanced outcoupling of organic light-emitting devices with an embedded low-index grid," Appl. Phys. Lett. 94, 163302 (2009).

[30] S.-Y. Kim, and J.-J. Kim, "Outcoupling efficiency of organic light emitting diodes and the effect of ITO thickness," Org. Electron. 11, 1010-1015 (2010). 